\title{
Publisher Correction: Aerodynamic generation of electric fields in turbulence laden with charged inertial particles
}

\author{
M. Di Renzo (1) ${ }^{1,2} \&$ J. Urzay ${ }^{1}$
}

Correction to: Nature Communications https://doi.org/10.1038/s41467-018-03958-7; published online 26 April 2018

The original version of this Article contained an error in the last sentence of the second paragraph of the 'Atmospheric rarefaction effects' section of the Results, which incorrectly read 'The other one emulates the rarefied, $\mathrm{CO}_{2}$-rich $\mathrm{Martian}$ atmosphere $\left(\mu_{\delta}=\right.$ $1.3 \times 10^{-5} \mathrm{~N} \mathrm{~s} \mathrm{~m}^{-2}$ ) at $6.9 \mathrm{mbar}$ and $210 \mathrm{~K}$, which gives $\rho_{\delta^{\star}}=1.6 \times 10^{-12} \mathrm{~kg} \mathrm{~m}^{-3}$.' The correct version states ' $\rho_{\delta^{\star}}=1.6 \times 10^{-2} \mathrm{~kg} \mathrm{~m}$ -3 ' in place of ' $\rho_{\delta^{*}}=1.6 \times 10^{-12} \mathrm{~kg} \mathrm{~m}^{-3}$ '. This has been corrected in both the PDF and HTML versions of the Article.

Published online: 21 May 2018

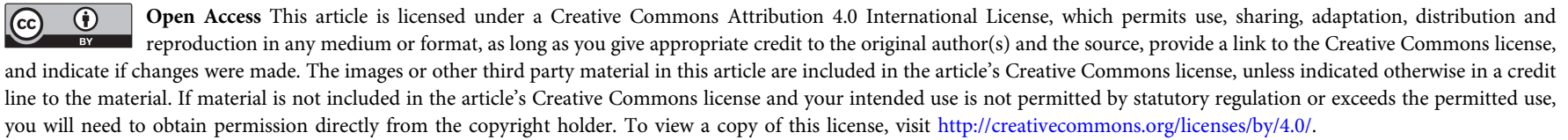

(C) The Author(s) 2018

\footnotetext{
${ }^{1}$ Center for Turbulence Research, Stanford University, Stanford, CA 94305, USA. ${ }^{2}$ Dipartimento di Meccanica, Matematica e Management, Politecnico di Bari, Bari 70125, Italy. Correspondence and requests for materials should be addressed to J.U. (email: jurzay@stanford.edu)
} 